\title{
Stability islands in domains of separatrix crossings in slow-fast Hamiltonian systems
}

\author{
Anatoly Neishtadt ${ }^{1}$, Carles Simón ${ }^{2}$ Dmitri Treschev ${ }^{3}$, and Alexei Vasiliev ${ }^{1}$ \\ 1 Space Research Institute, Profsoyuznaya 84/32, Moscow 117997, Russia, \\ 2 Departament de Matemàtica Aplicada i Anàlisi, Univ. de Barcelona, \\ Gran Via, 585, Barcelona 08007, Spain, \\ 3 V.A. Steklov Mathematical Institute, Gubkina 8, Moscow 119991, Russia.
}

\begin{abstract}
We consider a 2 d.o.f. Hamiltonian system with one degree of freedom corresponding to fast motion and the other corresponding to slow motion. The ratio of the time derivatives of slow and fast variables is of order $0<\varepsilon \ll 1$. At frozen values of the slow variables there is a separatrix on the phase plane of the fast variables and there is a region in the phase space (the domain of separatrix crossings) where the projections of phase points onto the plane of the fast variables repeatedly cross the separatrix in the process of evolution of the slow variables. Under a certain symmetry condition we prove existence of many (of order $1 / \varepsilon$ ) stable periodic trajectories in the domain of the separatrix crossings. Each of these trajectories is surrounded by a stability island whose measure is estimated from below by a value of order $\varepsilon$. Thus, the total measure of the stability islands is estimated from below by a value independent of $\varepsilon$. The proof is based on an analysis of the asymptotic formulas for the corresponding Poincaré map.
\end{abstract}

\section{Introduction}

Many problems in the theory of charged particles' motion, the theory of propagation of short-wave excitations, and in celestial mechanics can be reduced to the analysis of 2 d.o.f. Hamiltonian systems with fast and slow variables [1, 2, 3]. One degree of freedom corresponds to fast variables, and the other corresponds to slow variables. The ratio of the time derivatives of slow and fast variables is of order $0<\varepsilon \ll 1$. To describe the dynamics in such systems one can use the adiabatic approximation constructed as follows.

Consider the fast system, i.e. the system for the fast variables at frozen values of the slow variables. This is a 1 d.o.f. Hamiltonian system involving the slow variables as parameters. Assume that for a range of values of the slow variables there is a region filled with closed trajectories on the phase portrait of the fast system. Then one can introduce "action-angle" variables in the fast system [4]. The "action" variable of the fast system is an adiabatic invariant (i.e. an approximate first integral) of the complete system: its value oscillates with amplitude $\sim \varepsilon$ on time periods of order $\sim 1 / \varepsilon$ along a phase trajectory. To 
describe approximately the variation of the slow variables, one should average the rates of their variation over the "angle" variable of the fast motion. This approximation of the real motion is called the adiabatic approximation [5]. The obtained 1 d.o.f. Hamiltonian system for the slow variables, involving the "action" variable of fast motion as a parameter, is called the slow system.

Assume that for some range of values of the slow variables the "action" variable phase trajectories of the slow system are closed. Then in this range the motion can be described with V.I.Arnold's theorem on perpetual adiabatic invariance [6]: the "action" value along a trajectory perpetually undergoes only small, of order $\varepsilon$, oscillations (under a certain generality condition). The considered region of the phase space is filled, up to a small residual set, with invariant tori, which are $\varnothing(\varepsilon)$-close to the invariant tori of the system of the adiabatic approximation.

We shall consider cases in which there are separatrices on the fast system's phase portrait (see Figure 11), and there is a region in the phase space (the domain of separatrix crossings) where the projections of phase points onto the plane of the fast variables repeatedly cross the separatrix in the process of evolution of the slow variables. In this case the above described construction of the adiabatic approximation needs some modification, and the above formulated assertions on the adiabatic approximation accuracy are not applicable.

We shall assume that a certain symmetry condition is valid: the areas inside the separatrix loops in Figure 1 are equal.

The main result of the present paper is as follows. Under certain generality conditions in the domain of separatrix crossings on every energy level there exist many, of order $1 / \varepsilon$, stable periodic trajectories of period $\sim 1 / \varepsilon$. Each of these trajectories is surrounded by a stability island, and the measure of this island is estimated from below by a value of order $\varepsilon$. Thus, the total measure of the stability islands is estimated from below by a quantity which is independent of $\varepsilon$. A stability island is a domain on an energy level bounded by a two-dimensional invariant torus. A stability island contains a discrete family of invariant tori contractible to the periodic trajectory. Introduce a "modified action" equal to the "action" for points inside the separatrix loops in Figure 1, and equal to one half of the "action" for the other points. This "modified action" is a perpetual adiabatic invariant of the motion inside a stability island: its value along a phase trajectory perpetually undergoes only oscillations with amplitude of order $\varepsilon$. Therefore, the stability islands are also islands of perpetual adiabatic invariance.

We note that the existence of stability islands with total measure that is not small with $\varepsilon$ in the domain of separatrix crossings is quite unexpected. Visually, in many problems, this domain looks like a region of dynamical chaos (see [7, 8]). It was shown in [9] that a single stability island cannot have a measure larger than $\varnothing(\varepsilon)$.

The existence of stability islands with total measure not small with $\varepsilon$ in the domain of separatrix crossings was established in [10, 11] in the case of a Hamiltonian system with one degree of freedom and the Hamiltonian function slowly periodically depending on time. Here we generalize this result to 2 d.o.f. systems. Like in [10, 11], the proofs are based on the study of asymptotic formulas for the corresponding Poincaré map. In [10, 11] these formulas were constructed with the use of asymptotic expressions for the jump of the adiabatic invariant at a separatrix crossing in systems with one and a half d.o.f. [12, 13, 14] and for the variation of the "angle" variable between separatrix crossings in such systems [15]. Below we use analogous formulas for systems with two d.o.f. [16, 17]. To find 
linearly stable periodic trajectories we look for linearly stable fixed points of the Poincaré map. The results on Lyapunov stability of the periodic trajectories (the fixed points) and on the existence of invariant tori surrounding the periodic trajectories (invariant curves around the fixed points) are provided by the Kolmogorov-Arnold-Moser (KAM) theory. An example of application of the present theory to the motion of a charged particle in the parabolic model of magnetic field in the Earth magnetotail is given in [18].

\section{Formulation of the results}

Consider a two degrees of freedom Hamiltonian system with Hamiltonian $H=$ $H(p, q, y, x)$, where $q, \varepsilon^{-1} x$ are coordinates, and $p, y$ are canonically conjugated momenta, $\varepsilon>0$ is a small parameter, $H \in C^{\infty}$. The corresponding equations of motion are:

$$
\dot{p}=-\frac{\partial H}{\partial q}, \quad \dot{q}=\frac{\partial H}{\partial p}, \quad \dot{y}=-\varepsilon \frac{\partial H}{\partial x}, \quad \dot{x}=\varepsilon \frac{\partial H}{\partial y} .
$$

The variables $p, q$ are called fast variables, while $y, x$ are called slow variables. The Hamiltonian system for $p, q$ at $(y, x)=$ const is called fast (or unperturbed) system.

Assume that at all considered values of the slow variables the phase portrait of the fast system looks as in figure 1. On this phase portrait, there exist a non-degenerate saddle point $C$ and separatrices $l_{1}, l_{2}$. These separatrices divide the $(p, q)$-plane into domains $G_{i}=G_{i}(y, x), i=1,2,3$. Denote the value of $H$ at the saddle point as $h_{s}=h_{s}(y, x)$, and introduce $E=E(p, q, y, x)=H-h_{s}$. On the separatrices $E=0$. We suppose that $E>0$ in $G_{3}$ and $E<0$ in $G_{1}$ and $G_{2}$. The areas of the domains $G_{1}, G_{2}$ are $S_{1}, S_{2}$ respectively, and $S_{3}=S_{1}+S_{2} ; S_{i}=S_{i}(y, x)$. We will make the following symmetry assumption:

A) The areas of $G_{1}, G_{2}$ are equal: $S_{1}(y, x)=S_{2}(y, x)=S(y, x)$.

Put $\Theta=\Theta(y, x)=\left\{S, h_{s}\right\}$, where $\{\cdot, \cdot\}$ is the Poisson bracket with respect to the variables $(y, x):\{f, g\}=f_{x}^{\prime} g_{y}^{\prime}-f_{y}^{\prime} g_{x}^{\prime}$.

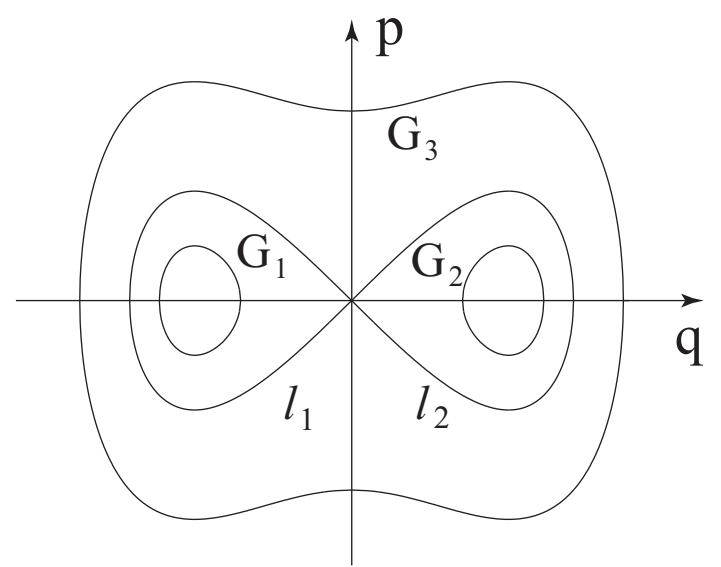

Figure 1: Phase portrait of the fast system.

In the unperturbed system, one can introduce canonical "action-angle" variables $(I, \varphi)$ separately in each $G_{i}$. The "action" variable $I=I(p, q, y, x)$ in the unperturbed system involves $y, x$ as parameters: $I=A /(2 \pi)$, where $A$ is the area on the phase portrait of the fast system surrounded by a phase trajectory passing through the point $(p, q)$. For all $p, q$ 
on the Hamiltonian level line $\{p, q: H(p, q, y, x)=h\}$ in the domain $G_{i}$ the function $I$ takes the same value $I_{i}(h, y, x)$.

Introduce the continuous function $\hat{I}(p, q, y, x)$ as follows:

$$
\begin{aligned}
& \hat{I}(p, q, y, x)=I(p, q, y, x), \quad \text { if }(p, q) \in G_{1,2}(y, x) \text {; } \\
& \hat{I}(p, q, y, x)=I(p, q, y, x) / 2, \quad \text { if }(p, q) \in G_{3}(y, x) \text {; } \\
& \hat{I}(p, q, y, x)=S(y, x) /(2 \pi), \quad \text { if }(p, q) \in l_{1} \cup l_{2} \cup C \text {. }
\end{aligned}
$$

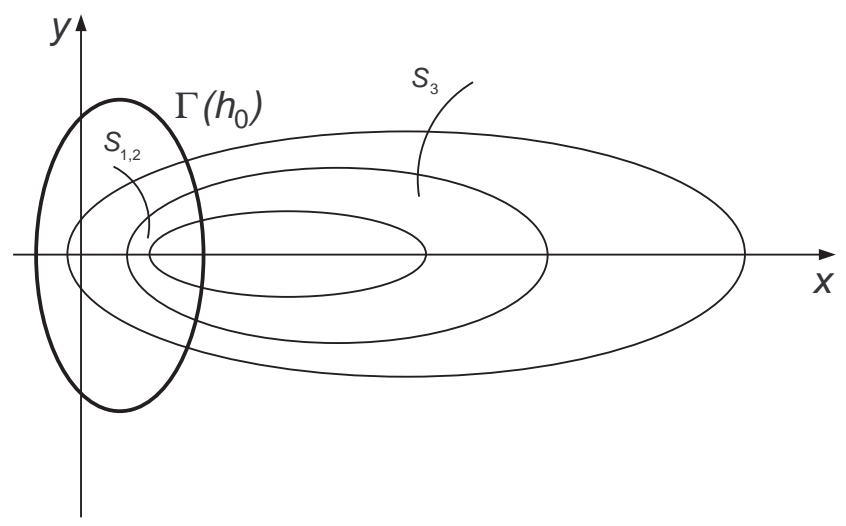

Figure 2: Schematic picture of motion on the slow plane.

The function $\hat{I}$ is an adiabatic invariant of the perturbed system. Far from the separatrix of the unperturbed system, its value along a phase trajectory is preserved with an accuracy of order $\varepsilon$ on time intervals of order $1 / \varepsilon[6]$. When a phase point crosses a narrow neighborhood of the separatrix, $\hat{I}$ varies by a value of order $\varnothing(\varepsilon)$ for the majority of initial conditions [16]. The approximation $\hat{I}=$ const along a phase trajectory is called adiabatic. In this approximation the dynamics of the slow variables is described by a Hamiltonian system with Hamiltonian $H_{0}(\hat{I}, y, x)$, where $H_{0}$ is the function $H$ expressed in terms of $\hat{I}, y, x$. Consider the dynamics on an energy level $H=h_{0}$. On the phase plane of the slow variables $(y, x)$, the separatrix is represented by a curve $\Gamma\left(h_{0}\right)=\left\{y, x: h_{s}(y, x)=h_{0}\right\}$ (see figure 2). This curve is called the uncertainty curve [3]. It divides the plane into two domains: $D_{3}$, corresponding to $G_{3}$ on the fast plane, and the other, $D_{1,2}$, corresponding to $G_{1}$ and $G_{2}$. In the adiabatic approximation, a phase point on the slow plane moves along level lines $I\left(h_{0}, y, x\right)=$ const. As the area inside the separatrix loops varies, a phase trajectory can cross the separatrix. Fix $\nu=1$ or $\nu=2$. Let $B_{\nu}\left(\hat{I}_{0}\right)$ be the adiabatic trajectory with $\hat{I}=\hat{I}_{0}$ on the plane of the slow variables for phase points passing through $G_{\nu}$ :

$$
B_{\nu}\left(\hat{I}_{0}\right)=\left\{y, x: I_{3}\left(h_{0}, y, x\right)=2 \hat{I}_{0},(y, x) \in D_{3} ; I_{\nu}\left(h_{0}, y, x\right)=\hat{I}_{0},(y, x) \in D_{1,2}\right\}
$$

Let us make the following assumption.

B) There exists $\hat{I}_{*}$ such that the curve $B_{\nu}\left(\hat{I}_{*}\right)$ is closed and intersects the uncertainty curve $\Gamma\left(h_{0}\right)$ at two points; at these points $\Theta(x, y) \neq 0$. On $B_{\nu}$ there are no stationary points of the Hamiltonian $H_{0}$.

If assumption B) is valid both for $\nu=1$ and $\nu=2$ (with the same $\hat{I}_{*}$ ), then in the adiabatic approximation, the projection of the motion with $\hat{I}=\hat{I}_{0}$ onto the slow plane looks as follows. In the domain corresponding to $G_{3}$, a phase point moves along the curve $I_{3}(h, y, x)=2 \hat{I}_{*}$. After crossing $\Gamma\left(h_{0}\right)$, it follows one of the curves $I_{\nu}\left(h_{0}, y, x\right)=\hat{I}_{*}[$ in 
general, these two curves are different]. Then the phase point crosses $\Gamma\left(h_{0}\right)$ again and continues its motion along $I_{3}\left(h_{0}, y, x\right)=2 \hat{I}_{*}$.

Assumption B) implies that there exists an interval $\Xi$ of values of $\hat{I}$, such that for all $\hat{I}_{0} \in D$ the curve $B_{\nu}\left(\hat{I}_{0}\right)$ has the property $\left.\mathrm{B}\right)$. In the exact system, multiple separatrix crossings occur in this interval of values of $\hat{I}$. Computer simulations demonstrate diffusion of the adiabatic invariant and chaotic dynamics in this region, see, e.g., [18].

The main result of the paper can be formulated as follows.

Theorem. Under additional generality conditions, in the domain $\{p, q, y, x: \hat{I} \in \Xi\}$ on the energy level $H=h_{0}$ there exist more than $C_{1}^{-1} / \varepsilon$ stable periodic trajectories of system (1). Each of these trajectories is surrounded by an invariant torus bounding a domain of (3-dimensional) phase volume greater than $C_{2}^{-1} \varepsilon$. Inside each of these domains the variation of $\hat{I}$ is smaller than $C_{3} \varepsilon$.

Corollary. The total measure of these domains is larger than $C_{4}^{-1}=C_{1}^{-1} C_{2}^{-1}$.

Here $C_{i}$ are positive constants independent of $\varepsilon$. The generality conditions mentioned in the theorem are formulated below, in Sections 6 and 7.

The domains bounded by invariant tori are domains (or islands) of perpetual adiabatic invariance. They are also called stability islands. Projected on the phase plane of the fast variables $(p, q)$, the motion along the stable periodic trajectories under consideration looks as follows. A phase point starts moving in region $G_{3}$, then crosses the separatrix and gets into region $G_{\nu}$, then crosses the separatrix again, comes back into $G_{3}$, and returns to its starting point.

The stable periodic trajectories mentioned in the theorem correspond to stable stationary points of the return map generated by the original system. This map is written in the principal approximation in Section 5.

Remark. The result of the theorem is valid also if the phase portrait of the fast system is of the kind shown in figure 1 but not for all $x, y$. It is enough to require that the system has such a phase portrait at the time of separatrix crossing calculated in the adiabatic approximation.

\section{$3 \quad$ Adiabatic and improved adiabatic approximations}

In the fast system, action-angle variables $I, \varphi \bmod 2 \pi$ are introduced separately in each of the $G_{i}$ domains by a canonical transformation of variables. The corresponding generating function $W(I, q, y, x)$ contains $y, x$ as parameters [for brevity, we omit subscripts $i$ ]. We take this function in the form

$$
W(I, q, y, x)=\int_{q_{0}(I, y, x)}^{q} \mathcal{P}\left(I, q^{\prime}, y, x\right) \mathrm{d} q^{\prime},
$$

where $\mathcal{P}$ is the value of the $p$-variable along the trajectory with the prescribed value of the action $I$, and $q_{0}(I, y, x)$ defines a curve in $G_{i}$ transversal to the phase trajectories. In the new variables the Hamiltonian has the form $H=H_{0}(I, y, x)$.

Now make a canonical transformation of variables $(p, q, y, x) \mapsto(\bar{I}, \bar{\varphi}, \bar{y}, \bar{x})$ with the generating function $\bar{y} \varepsilon^{-1} x+W(\bar{I}, q, \bar{y}, x)$. The canonically conjugated pairs of variables are $(\bar{I}, \bar{\varphi})$ and $\left(\bar{y}, \varepsilon^{-1} \bar{x}\right)$. Formulas for the transformation of variables are:

$$
\bar{\varphi}=\partial W / \partial \bar{I}, \quad p=\partial W / \partial q, \quad \bar{x}=x+\varepsilon \partial W / \partial \bar{y}, \quad y=\bar{y}+\varepsilon \partial W / \partial x .
$$


In the new variables, the Hamiltonian $H$ has the form

$$
H=H_{0}(\bar{I}, \bar{y}, \bar{x})+\varepsilon H_{1}(\bar{I}, \bar{\varphi}, \bar{y}, \bar{x})+\emptyset\left(\varepsilon^{2}\right),
$$

where

$$
H_{1}=\frac{\partial H}{\partial y} \frac{\partial W}{\partial x}-\frac{\partial H_{0}}{\partial x} \frac{\partial W}{\partial \bar{y}} .
$$

In the adiabatic approximation, the dynamics is described by the Hamiltonian $H_{0}$. In this approximation $\bar{I}=$ const along phase trajectories.

One can also construct a canonical, close to the identity, transformation of variables $(\bar{I}, \bar{\varphi}, \bar{y}, \bar{x}) \mapsto(J, \psi, \hat{y}, \hat{x})$ in order to make the terms of order $\varepsilon$ in the Hamiltonian independent of the phase (see, for example, [17]). In the new variables, the Hamiltonian takes the form:

$$
\mathcal{H}=H_{0}(J, \hat{y}, \hat{x})+\varepsilon \mathcal{H}_{1}(J, \hat{y}, \hat{x})+\varepsilon^{2} \mathcal{H}_{2}(J, \psi, \hat{y}, \hat{x}, \varepsilon), \quad \mathcal{H}_{1}=\left\langle H_{1}\right\rangle,
$$

where the brackets denote averaging with respect to $\bar{\varphi}$.

In the improved adiabatic approximation, the dynamics is described by the Hamiltonian $H_{0}(J, Y, X)+\varepsilon \mathcal{H}_{1}(J, Y, X)$. In this approximation $J$ is an integral of motion. With an accuracy of order $\varepsilon^{2}$, the following formula for $J$ is valid (see [16]):

$$
\begin{array}{r}
J=J(p, q, y, x)=I+\varepsilon u(p, q, y, x), \\
u=\frac{1}{4 \pi}\left[\int_{0}^{T}\left(\frac{\partial E}{\partial y} \int_{0}^{t} \frac{\partial E}{\partial x} \mathrm{~d} \sigma\right) \mathrm{d} t-\int_{0}^{T}\left(\frac{\partial E}{\partial x} \int_{0}^{t} \frac{\partial E}{\partial y} \mathrm{~d} \sigma\right) \mathrm{d} t\right] \\
+\frac{1}{2 \pi} \int_{0}^{T}\left(\frac{T}{2}-\sigma\right)\left\{E, h_{s}\right\} \mathrm{d} \sigma .
\end{array}
$$

The integrals here are calculated along a phase trajectory of the fast system passing through the point $(p, q) ; \sigma$ is the time of motion along this trajectory starting from this point, $T$ is the period of motion. The function $J$ is the improved adiabatic invariant. In the complete system far from separatrices its value along a phase trajectory is constant with an accuracy of order $\varepsilon^{2}$ on time intervals of order $\varepsilon^{-1}$. In what follows, it is convenient to use the function $\hat{J}(p, q, y, x)$ equal to $J$ in the domains $G_{1}, G_{2}$ and equal to $J / 2$ in the domain $G_{3}$.

\section{Description of the separatrix crossing}

On the phase plane $\mathbb{P}^{s l}$ of the slow variables $(y, x)$, the separatrix is represented by a curve $\Gamma\left(h_{0}\right)$ (see figure 2). Let $B_{\nu}(\hat{I}) \in \mathbb{P}^{s l}$ be a curve (see (2) ) corresponding to $\hat{I}$ such that $\hat{I} \in \Xi$. According to B), $B_{\nu}(\hat{I})$ is divided by $\Gamma\left(h_{0}\right)$ into two parts. Consider two segments $\mathcal{S}_{3}$ and $\mathcal{S}_{\nu}$ on $\mathbb{P}^{s l}$ separated by $\Gamma\left(h_{0}\right)$, such that each of the segments crosses transversely all the curves $B_{\nu}(\hat{I})$ with $\hat{I} \in \Xi$ at a distance of order 1 from $\Gamma\left(h_{0}\right)$. Points on the fast plane corresponding to $\mathcal{S}_{3}$ belong to $G_{3}$, and the points corresponding to $\mathcal{S}_{\nu}$ belong to $G_{\nu}$. Fix an interval $\Xi_{0} \in \Xi$, assuming that the endpoints of $\Xi_{0}$ and $\Xi$ are different.

Let the motion start at $t=0$ in a point $M^{(0)}\left(p^{(0)}, q^{(0)}, y^{(0)}, x^{(0)}\right)$, such that $H\left(p^{(0)}, q^{(0)}, y^{(0)}, x^{(0)}\right)=h_{0}$ and on the plane $\mathbb{P}^{s l}\left(\hat{y}^{(0)}, \hat{x}^{(0)}\right) \in \mathcal{S}_{3}$. Hence, $\left(p^{(0)}, q^{(0)}\right) \in$ 
$G_{3}\left(y^{(0)}, x^{(0)}\right)$. Let $\hat{I}=\hat{I}^{(0)}, \hat{J}=\hat{J}^{(0)}, \psi=\psi^{(0)}$ at this point; $\hat{I}^{(0)} \in \Xi_{0}$. In the adiabatic approximation the trajectory of the slow variables is $B\left(\hat{I}^{(0)}\right)$, the motion is described by a Hamiltonian system with Hamiltonian $H_{0}\left(\hat{I}^{(0)}, y, x\right)$. Let $\left(x_{-}, y_{-}\right)$and $\left(x_{+}, y_{+}\right)$denote the points of intersection of $B\left(\hat{I}^{(0)}\right)$ and $\Gamma\left(h_{0}\right)$; the sign "-" corresponds to passage from $G_{3}$ to $G_{\nu}$ and the sign "+" corresponds to passage from $G_{\nu}$ to $G_{3}$. Below $\tau_{-}$and $\tau_{+}$ denote the slow time moments of separatrix crossing in this approximation. In the exact system, in the process of evolution the phase point on $\mathbb{P}^{s l}$ approaches the curve $\Gamma\left(h_{0}\right)$, and accordingly, on the plane $(p, q)$ it approaches the separatrix. We are interested in the dynamics of phase points that are being captured into $G_{\nu}$ after the separatrix crossing. For such a phase point, the first crossing of the $C q$-axis in $G_{\nu}$ (see figure 1) occurs near $C$ at time $\tau=\tau_{-}+\varnothing(\varepsilon)$. [We assume for simplicity that coordinate axes in figure 1 are the principal axes of the saddle point.] Denote the value of $E$ at the point of this crossing as $h^{(0)}$. We introduce $\eta^{(0)}=1-\left|h^{(0)} / \varepsilon \Theta_{-}\right|$. Here $\Theta_{-} \equiv \Theta\left(y_{-}, x_{-}\right)>0$.

After the separatrix crossing the phase point in the plane of slow variables $(\hat{y}, \hat{x})$ moves towards $\mathcal{S}_{\nu}$. When it crosses $\mathcal{S}_{\nu}$, the projection of the phase point onto the fast plane is deep inside region the $G_{\nu}$. Denote the value of $\hat{J}$ at this time moment as $\hat{J}^{(1)}$.

Then the phase point starts again approaching the separatrix. At $\tau=\tau_{+}+\varnothing(\varepsilon)$ the phase point crosses $C q$-axis in $G_{\nu}$ near the point $C$ for the last time before entering $G_{3}$. Denote the value of $E$ at the point of this crossing as $h^{(1)}$. We introduce $\eta^{(1)}=\left|h^{(1)} / \varepsilon \Theta_{+}\right|$. Here $\Theta_{+} \equiv \Theta\left(y_{+}, x_{+}\right)<0$.

After crossing $\Gamma\left(h_{0}\right)$, the projection of the phase point onto the plane $(\hat{y}, \hat{x})$ crosses again the segment $\mathcal{S}_{3}$. Let $\hat{J}^{(2)}, \psi^{(2)}$ denote the values of $\hat{J}, \psi$ at this time moment. Then the phase point approaches the separatrix again, crosses it and gets captured into $G_{l}, l=1$ or $l=2$. Let $E=h^{(2)}$ at the first crossing of the $C q$-axis in $G_{l}$ near $C$. This crossing occurs at time $\tau=\tau_{-}+T_{0}+\varnothing(\varepsilon)$, where $T_{0}$ is the slow time period of motion along the trajectory $\hat{I}=\hat{I}^{(0)}$ in the adiabatic approximation. We introduce $\eta^{(2)}=1-\left|h^{(2)} / \varepsilon \Theta_{-}\right|$. We are interested in the dynamics of the phase points for which $l=\nu$.

\section{The return map}

In the energy level $H=h_{0}$ the segment $\mathcal{S}_{3}$ is represented by a piece of a two-dimensional surface $\left\{p, q, y, x: H(p, q, y, x)=h_{0},(\hat{y}, \hat{x}) \in \mathcal{S}_{3}\right\}$. This piece can be parametrized by variables $\hat{J}, \psi$. The corresponding Poincaré return map $M:\left(\hat{J}^{(0)}, \psi^{(0)}\right) \rightarrow\left(\hat{J}^{(2)}, \psi^{(2)}\right)$ produced by trajectories that pass through $G_{\nu}$ is symplectic. Its stable stationary points correspond to stable periodic orbits of the original problem, of period approximately equal to $T_{0} / \varepsilon$. It is convenient to study these stationary points using a different set of variables, namely to consider the map $\hat{M}:\left(\hat{J}^{(0)}, \eta^{(0)}, \nu\right) \rightarrow\left(\hat{J}^{(2)}, \eta^{(2)}, l\right)$.

The map $\hat{M}$ is the composition of two maps: $\hat{M}=M^{(2)} \circ M^{(1)}$,

$$
M^{(1)}:\left(\hat{J}^{(0)}, \eta^{(0)}, \nu\right) \rightarrow\left(\hat{J}^{(1)}, \eta^{(1)}, \nu\right), \quad M^{(2)}:\left(\hat{J}^{(1)}, \eta^{(1)}, \nu\right) \rightarrow\left(\hat{J}^{(2)}, \eta^{(2)}, l\right) .
$$

The results of [16, 17] give the following formulas for $M^{(k)}$. Suppose that

$$
\eta^{(0)}, \eta^{(1)}, \eta^{(2)} \in\left[c_{1}^{-1}, 1-c_{1}^{-1}\right] .
$$

Then 


$$
\begin{aligned}
\hat{J}^{(1)}= & \hat{J}^{(0)}-(2 \pi)^{-1} \varepsilon a_{-} \Theta_{-} \ln \left(2 \sin \pi \eta^{(0)}\right)+(2 \pi)^{-1} \varepsilon\left(d_{\nu,-}-d_{3,-} / 2\right)+ \\
& (2 \pi)^{-1} \varepsilon \Theta_{-}\left(\eta^{(0)}-1 / 2\right)\left(b_{\nu,-}-b_{3,-} / 2\right)+O\left(\varepsilon^{3 / 2} \ln \varepsilon\right), \\
\eta^{(1)}= & \left\{\eta^{(0)}+\varepsilon^{-1} \Phi_{1}^{(\nu)}\left(\hat{J}^{(1)}, \varepsilon\right)+O\left(\varepsilon^{1 / 3} \ln ^{-1 / 3} \varepsilon\right)\right\}, \\
\hat{J}^{(2)}= & \hat{J}^{(1)}-(2 \pi)^{-1} \varepsilon a_{+} \Theta_{+} \ln \left(2 \sin \pi \eta^{(1)}\right)-\varepsilon(2 \pi)^{-1}\left(d_{\nu,+}-d_{3,+} / 2\right)+ \\
& (2 \pi)^{-1} \varepsilon \Theta_{+}\left(1 / 2-\eta^{(1)}\right)\left(b_{\nu,+}-b_{3,+} / 2\right)+O\left(\varepsilon^{3 / 2} \ln \varepsilon\right), \\
\eta^{(2)=} & \left\{\eta^{(1)}+\varepsilon^{-1} \Phi_{2}\left(\hat{J}^{(2)}, \varepsilon\right)+O\left(\varepsilon^{1 / 3} \ln ^{-1 / 3} \varepsilon\right)\right\}, \\
& l=\nu, \quad \text { if } \quad 0<\left\{\left(\varepsilon^{-1} \Phi_{2}\left(\hat{J}^{(2)}, \varepsilon\right)+\eta^{(1)}\right) / 2\right\}<1 / 2, \\
& l \neq \nu, \quad \text { if } \quad 1 / 2<\left\{\left(\varepsilon^{-1} \Phi_{2}\left(\hat{J}^{(2)}, \varepsilon\right)+\eta^{(1)}\right) / 2\right\}<1 .
\end{aligned}
$$

Here $\{\cdot\}$ denotes the fractional part,

$$
\begin{array}{r}
a_{ \pm}=a\left(y_{ \pm}, x_{ \pm}\right), \quad b_{j, \pm}=b_{j}\left(y_{ \pm}, x_{ \pm}\right), \quad d_{j, \pm}=d_{j}\left(y_{ \pm}, x_{ \pm}\right), \\
j=1,2,3, \quad b_{3}=b_{1}+b_{2}, \quad d_{3}=d_{1}+d_{2} .
\end{array}
$$

For $a$ one has $a=1 / \sqrt{-g}$, where $g$ is the Hessian of $E$ at the point $C$. The values $d_{1,2}$ are defined as the main terms of the expansions (cf. [16])

$$
2 \pi u(p, q, y, x)=d_{j}+\varnothing(\sqrt{|E|} \ln |E|),
$$

where $u$ is given by (9), and the point $(p, q)$ is on the $C q$-axis (figure 11) in $G_{j}$ near $C$. Values $b_{j}$ come from the expansion of the period of motion in $G_{j}, j=1,2$ near the separatrix:

$$
T_{j}=-a \ln |E|+b_{j}+\varnothing(E \ln |E|)
$$

In (12), (14),

$$
\begin{aligned}
\Phi_{1}^{(\nu)}(\hat{J}, \varepsilon) & =\frac{1}{2 \pi} \int_{\tau_{-}^{(\nu)}}^{\tau_{+}^{(\nu)}}\left(\omega_{0}^{(\nu)}\left(\hat{J}, Y_{\nu}(\tau), X_{\nu}(\tau)\right)+\varepsilon \omega_{1}^{(\nu)}\left(\hat{J}, Y_{\nu}(\tau), X_{\nu}(\tau)\right)\right) \mathrm{d} \tau \\
\Phi_{2}(\hat{J}, \varepsilon) & =\frac{1}{\pi} \int_{\tau_{+}^{(3)}}^{\tau_{-}^{(3)}}\left(\omega_{0}^{(3)}\left(2 \hat{J}, Y_{3}(\tau), X_{3}(\tau)\right)+\varepsilon \omega_{1}^{(3)}\left(2 \hat{J}, Y_{3}(\tau), X_{3}(\tau)\right)\right) \mathrm{d} \tau
\end{aligned}
$$

Here $\omega_{0}^{(j)}$ and $\omega_{1}^{(j)}$ are defined as $\omega_{0}^{(j)}=\partial H_{0} / \partial J, \omega_{1}^{(j)}=\partial \mathcal{H}_{1} / \partial J$, with $H_{0}, \mathcal{H}_{1}$ calculated in the region $G_{j}$, and $\left(Y_{j}, X_{j}\right)$ is a solution of the Hamiltonian system with Hamiltonian $\hat{H}=H_{0}(J, y, x)+\varepsilon H_{1}(J, y, x)$ on the energy level $\hat{H}=h_{0}$. The values $\tau_{ \pm}^{(j)}$ are the slow time moments when the phase point corresponding to this solution arrives to the separatrix. At these moments $S_{j}(Y, X)=2 \pi J$. [One can take any of such solutions, they differ by a time shift which does not change the value of the integrals in (17), (18)]. Conditions (10) are understood inductively: if $\eta^{(0)} \in\left[c_{1}^{-1}, 1-c_{1}^{-1}\right]$, and $\eta^{(1)}$, found according to (11), (12) lies in segment $\left[c_{1}^{-1}, 1-c_{1}^{-1}\right]$, then (12) is valid, and analogously for $\eta^{(2)}$ and expressions (13), (14). 
Equations (11), (13) follow directly from the formula for the jump of the adiabatic invariant at a separatrix [16] for the case when the areas of domains $G_{1}$ and $G_{2}$ are equal. Equation (12) follows directly from the formula for phase change between separatrix crossings [17]. Equations (14)-(16) follow from the result of [17] in the following way.

Let $t_{+}, h_{+}$be the values of $t$ and $E$ when the phase point crosses the axis $C p$ near $C$ for the first time after exit from $G_{\nu}$. At time $t_{+}$the phase point is in $G_{3}$ on the positive or negative part of the axis $C p$. Let $h_{-}$be the value of $E$ when the phase point crosses the same part of $C p$ for the last time before exit from $G_{3}$. Introduce notations: $\bar{\zeta}=\frac{h_{+}}{2\left|\Theta_{+}\right|}, \zeta=\frac{h_{-}}{2\left|\Theta_{-}\right|}$. According to [17],

$$
\bar{\zeta}+\zeta=\frac{1}{2 \varepsilon} \Phi_{2}\left(\hat{J}^{(1)}, \varepsilon\right)+O\left(\varepsilon^{1 / 3} \ln ^{-1 / 3} \varepsilon\right) \bmod 1
$$

provided $\bar{\zeta}, \zeta \in\left[c_{2}^{-1}, 1-c_{2}^{-1}\right]$. The values $h^{(1)}$ (see Section 41) and $h_{+}$are related as $h_{+}=h^{(1)}+\varepsilon\left|\Theta_{+}\right|+\varnothing\left(\varepsilon^{3 / 2}\right)$ [16]. Therefore, $\bar{\zeta}=\frac{1}{2}\left(1-\eta^{(1)}\right)+\varnothing\left(\varepsilon^{1 / 2}\right)$. The values $h^{(2)}$ (see Section 44) and $h_{-}$are related as $h_{-}=h^{(2)}+2 \varepsilon \Theta_{-}+\varnothing\left(\varepsilon^{3 / 2}\right)$ if $l=\nu$, and as $h_{-}=h^{(2)}+\varepsilon \Theta_{-}+\varnothing\left(\varepsilon^{3 / 2}\right)$ if $l \neq \nu$. We have $l=\nu$ provided that $1 / 2<\zeta<1$. Consider this case. We obtain that $\zeta=\frac{1}{2}\left(1+\eta^{(2)}\right)+\varnothing\left(\varepsilon^{1 / 2}\right)$. From (19) we find

$$
\frac{1}{2}\left(1-\eta^{(1)}\right)+\frac{1}{2}\left(1+\eta^{(2)}\right)=\frac{1}{2 \varepsilon} \Phi_{2}\left(\hat{J}^{(2)}, \varepsilon\right)+O\left(\varepsilon^{1 / 3} \ln ^{-1 / 3} \varepsilon\right) \bmod 1 .
$$

Therefore,

$$
\eta^{(2)}=\eta^{(1)}+\frac{1}{\varepsilon} \Phi_{2}\left(\hat{J}^{(2)}, \varepsilon\right)+O\left(\varepsilon^{1 / 3} \ln ^{-1 / 3} \varepsilon\right) \bmod 2 .
$$

Then condition $1 / 2<\zeta<1$ implies equation (15).

Now consider the case $l \neq \nu$. In this case $0<\zeta<1 / 2$. We get that $\zeta$ and $\eta^{(2)}$ are related as $\zeta=\frac{1}{2} \eta^{(2)}+\varnothing\left(\varepsilon^{1 / 2}\right)$. From (19) we find

$$
\frac{1}{2}\left(1-\eta^{(1)}\right)+\frac{1}{2} \eta^{(2)}=\frac{1}{2 \varepsilon} \Phi_{2}\left(\hat{J}^{(2)}, \varepsilon\right)+O\left(\varepsilon^{1 / 3} \ln ^{-1 / 3} \varepsilon\right) \bmod 1 .
$$

Therefore,

$$
\eta^{(2)}=\eta^{(1)}+\frac{1}{\varepsilon} \Phi_{2}\left(\hat{J}^{(2)}, \varepsilon\right)-1+O\left(\varepsilon^{1 / 3} \ln ^{-1 / 3} \varepsilon\right) \bmod 2 .
$$

Combining (20) and (21) we obtain equation (14). Condition $0<\zeta<1 / 2$ implies (16).

\section{$6 \quad$ Linearly stable solutions}

\subsection{Equations for stable stationary points}

Linearly stable periodic solutions of the original problem of period approximately equal to $T_{0} / \varepsilon$ correspond to linearly stable fixed points of the map $\hat{M}$. These points are defined by the following set of conditions:

$$
\begin{array}{r}
\hat{J}^{(2)}=\hat{J}^{(0)}, \quad \eta^{(2)}=\eta^{(0)}, \quad l=\nu, \\
\left|\operatorname{tr} \hat{M}^{\prime}\right|<2,
\end{array}
$$

where $\hat{M}^{\prime}$ is the linearization of $\hat{M}$ at the fixed point. 
Put $\Phi_{1} \equiv \Phi_{1}^{(\nu)}$. Introduce also the following notations:

$$
\begin{array}{r}
\hat{J}^{(s)}=\varepsilon \xi^{(s)}, \quad s=0,1,2 ; \\
\varepsilon^{-1} \frac{d \Phi_{k}(\varepsilon \xi, \varepsilon)}{d \xi}=\gamma_{k}(\varepsilon \xi, \varepsilon), \quad \gamma_{k}(\varepsilon \xi, 0)=\gamma_{k}^{0}(\varepsilon \xi), \quad k=1,2 ; \\
a_{ \pm} \Theta_{ \pm}=\mp 2 \pi \alpha_{ \pm}, \quad d_{\nu, \pm}-d_{3, \pm} / 2=-2 \pi d_{ \pm}, \quad \Theta_{ \pm}\left(b_{\nu, \pm}-b_{3, \pm} / 2\right)=2 \pi b_{ \pm} .
\end{array}
$$

Suppose that $\left(\xi^{(0)}, \eta^{(0)}\right)=(\xi, \eta)$ correspond to a stationary point of the map $\hat{M}$. We rewrite equations (22) using formulas (10)-(14), notations (24), and neglecting terms $O\left(\varepsilon^{1 / 3} \ln ^{-1 / 3} \varepsilon\right)$

$$
\begin{array}{r}
\alpha_{-} \ln (2 \sin \pi \eta)+d_{-}-(\eta-1 / 2) b_{-}=\alpha_{+} \ln \left(2 \sin \pi \eta^{(1)}\right)+d_{+}+\left(1 / 2-\eta^{(1)}\right) b_{+}, \\
\eta^{(1)}-\eta+\gamma_{1}^{0} \alpha_{-} \ln (2 \sin \pi \eta)+\gamma_{1}^{0} d_{-}-\gamma_{1}^{0}\left(\eta-\frac{1}{2}\right) b_{-}=\varepsilon^{-1} \Phi_{1}(\varepsilon \xi, \varepsilon) \bmod 1 \\
\eta-\eta^{(1)}=\varepsilon^{-1} \Phi_{2}(\varepsilon \xi, \varepsilon) \bmod 1 \\
\eta^{(1)}+\varepsilon^{-1} \Phi_{2}(\varepsilon \xi, \varepsilon) \in(0,1) \bmod 2, \\
\eta, \eta^{(1)} \in\left(c_{1}^{-1}, 1-c_{1}^{-1}\right) .
\end{array}
$$

The stability condition $\left|\operatorname{tr} \hat{M}^{\prime}\right|<2$ can be written using (11)-(14) [we again neglect terms $\left.O\left(\varepsilon^{1 / 3} \ln ^{-1 / 3} \varepsilon\right)\right]$ :

$$
\begin{array}{r}
-4<Q<0, \\
Q=\left(u_{2}-u_{1}\right)\left(\gamma_{2}^{0}+\gamma_{1}^{0}\right)-u_{2} u_{1} \gamma_{2}^{0} \gamma_{1}^{0}, \\
u_{1}=\alpha_{-} \pi \cot \pi \eta-b_{-}, \quad u_{2}=\alpha_{+} \pi \cot \pi \eta^{(1)}-b_{+} .
\end{array}
$$

The exact set of conditions for a stable periodic solution differs from (25)-(29) by terms $O\left(\varepsilon^{1 / 3} \ln ^{-1 / 3} \varepsilon\right)$ that can be differentiated in $\varepsilon \xi, \eta, \eta^{(1)}$ without changing their smallness order. Below, we find non-degenerate solutions of system (25)-(29). According to the implicit function theorem, for small enough $\varepsilon$, each of these solutions corresponds to a solution of the exact system.

\subsection{Existence of stable stationary points}

We assume that the following generality conditions are valid.

H1. In $\Xi_{0}$ there exists an interval of values of $I$ such that

$$
\gamma_{1}^{0}(I) \cdot \gamma_{2}^{0}(I) \neq 0, \quad \frac{\mathrm{d}}{\mathrm{d} I}\left(\gamma_{1}^{0}(I) / \gamma_{2}^{0}(I)\right) \neq 0 .
$$

In what follows, we consider only those values of $\varepsilon \xi$ that belong to the interval defined by H1.

Equation (25) has the form

$$
F\left(\eta ; \alpha_{-}, b_{-}, d_{-}\right)=F\left(\eta^{(1)} ; \alpha_{+}, b_{+}, d_{+}\right)
$$

with $F(\eta ; \alpha, b, d)=\alpha \ln (2 \sin \pi \eta)+d-b(\eta-1 / 2), \eta \in(0,1), \alpha>0$. We have $F \rightarrow-\infty$ as $\eta \rightarrow 0^{+}$or $\eta \rightarrow 1^{-}$. Moreover, $F$ has a unique maximum $F_{\max }=m(\alpha, b, d)$ on the interval $0<\eta<1$. We put $m_{ \pm}=m\left(\alpha_{ \pm}, b_{ \pm}, d_{ \pm}\right)$. 


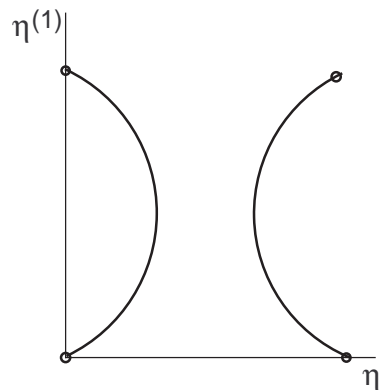

a)

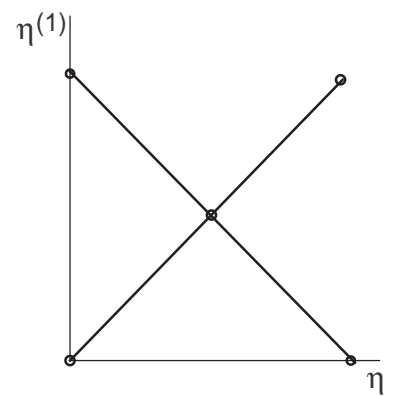

b)

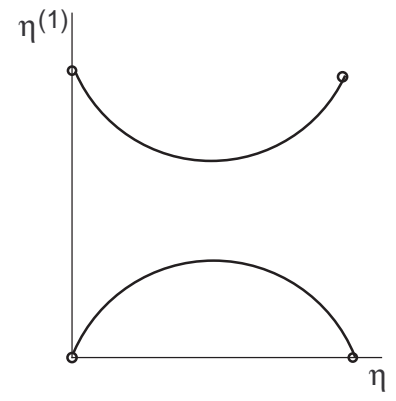

c)

Figure 3: Set of points $\left(\eta, \eta^{(1)}\right)$ satisfying (25) . a) $m_{-}>m_{+}$; b) $m_{-}=m_{+}$; c) $m_{-}<m_{+}$.

The set of points $\left(\eta, \eta^{(1)}\right) \in(0,1) \times(0,1)$ satisfying (25) is shown in figure 3. Consider for definiteness the case $m_{-}>m_{+}, \quad \gamma_{1}^{0} \gamma_{2}^{0}<0$. The other cases can be studied analogously.

Let in figure $3 A$ and $B$ have coordinates $(0,0)$ and $(0,1)$ respectively. Consider condition (30) on the curve $A B$. Near the point $A$ both $u_{1}$ and $u_{2}$ are large positive, and therefore $Q$ is large positive. Near the point $B$ both $u_{1}$ and $-u_{2}$ are large positive, and therefore $-Q$ is large positive. Therefore, on the segment $A B$ there is an interval $L=L(\varepsilon \xi)$ (or several intervals) where condition (30) is valid. If $c_{1}$ is sufficiently large, the relations (29) are also valid on $L(\varepsilon \xi)$.

Now we look for solutions of the system

$$
\begin{array}{rr}
\left(\eta, \eta^{(1)}\right) \in L(\varepsilon \xi), & \\
\eta^{(1)}-\eta+\gamma_{1}^{0} \alpha_{-} \ln (2 \sin \pi \eta)+\gamma_{1}^{0} d_{-}-\gamma_{1}^{0} b_{-}(\eta-1 / 2)=\varepsilon^{-1} \Phi_{1}(\varepsilon \xi, \varepsilon) & \bmod 1, \\
\eta-\eta^{(1)}=\varepsilon^{-1} \Phi_{2}(\varepsilon \xi, \varepsilon) & \bmod 2 .
\end{array}
$$

Relations (33)-(35) are equivalent to (25)-(31). Relation (28) holds because, according to (35), $\eta^{(1)}+\varepsilon^{-1} \Phi_{2}(\varepsilon \xi, \varepsilon)=\eta \bmod 2$ and $\eta \in(0,1)$. Validity of the other relations (25)-(31) follows from the definition of $L(\varepsilon \xi)$ and equations (34), (35).

System (33)-(35) can be interpreted geometrically as follows. Consider the curve $\Lambda(\varepsilon \xi) \subset \mathbb{T}^{2}=\left\{\left(s_{1} \bmod 1, s_{2} \bmod 2\right)\right\}$, which is the image of $L(\varepsilon \xi)$ under the mapping

$$
\left(\eta, \eta^{(1)}\right) \mapsto\left(s_{1}, s_{2}\right)=\left(\eta^{(1)}-\eta+\gamma_{1}^{0} \alpha_{-} \ln (2 \sin \pi \eta)+\gamma_{1}^{0} d_{-}-\gamma_{1}^{0}(\eta-1 / 2) b_{-}, \quad \eta-\eta^{(1)}\right)
$$

defined by the left hand sides of (34), (35). Consider also the point

$$
\varepsilon^{-1} \Phi(\varepsilon \xi, \varepsilon)=\left(\varepsilon^{-1} \Phi_{1}(\varepsilon \xi, \varepsilon) \bmod 1, \quad \varepsilon^{-1} \Phi_{2}(\varepsilon \xi, \varepsilon) \bmod 2\right)
$$

on $\mathbb{T}^{2}$. As $\xi$ varies, the curve $\Lambda(\varepsilon \xi)$ is moving slowly on the torus. At the same time, the point $\varepsilon^{-1} \Phi$ is moving fast, with its velocity vector $\gamma(\varepsilon \xi, \varepsilon)=\left(\gamma_{1}(\varepsilon \xi, \varepsilon), \gamma_{2}(\varepsilon \xi, \varepsilon)\right)$ varying slowly. Solutions of system (33)-(35) correspond to values of "time" $\xi$ when point $\varepsilon^{-1} \Phi(\varepsilon \xi, \varepsilon)$ crosses the curve $\Lambda(\varepsilon \xi)$.

It follows from assumption $\mathbf{H 1}$, that there exists $I_{c}$ such that the ratio $\gamma_{1}^{0}\left(I_{c}\right) / \gamma_{2}^{0}\left(I_{c}\right)$ is irrational. Then in the interval $\xi \in\left(I_{c}-c_{3}^{-1} / \varepsilon, I_{c}+c_{3}^{-1} / \varepsilon\right)$ on every segment of length $c_{4}$ of "time" $\xi$ the point $\varepsilon^{-1} \Phi(\varepsilon \xi, \varepsilon)$ crosses transversely the moving curve $\Lambda(\varepsilon \xi)$ at least once. Indeed, the point's trajectory is close to a dense winding of the torus with 
irrational angular coefficient $\gamma_{1}^{0}\left(I_{c}\right) / \gamma_{2}^{0}\left(I_{c}\right)$, and the curve $\Lambda\left(I_{c}\right)$ is transversal to the vector $\gamma^{0}\left(I_{c}\right)=\left(\gamma_{1}^{0}\left(I_{c}\right), \gamma_{2}^{0}\left(I_{c}\right)\right)$ at least at one point. [The latter is because $\Lambda\left(I_{c}\right)$ is not straight.]

Therefore, there exist more than $c_{3}^{-1} c_{4}^{-1} / \varepsilon-1>C_{1}^{-1} / \varepsilon$ intersection points. Each intersection point corresponds to a linearly stable stationary point of the return map. In Section 8 we obtain an asymptotic expression for the number of intersection points.

\section{$7 \quad$ Stability islands}

Let $\xi_{r}, \eta_{r}, \eta_{r}^{(1)}$ be one of the solutions of system (33)-(35), found in the previous section. One can rewrite the map $\hat{M}$ in variables $\tilde{\xi}=\xi-\xi_{r}, \eta$, expand its right hand sides in $\varepsilon$ supposing $\tilde{\xi} \sim 1$, and neglect terms $\varnothing\left(\varepsilon^{1 / 3} \ln ^{-1 / 3} \varepsilon\right)$. Thus, one obtains a map that does not contain $\varepsilon$ and depends on the parameter $I_{r}=\varepsilon \xi_{r}$. This map has a stationary linearly stable point $\tilde{\xi}=0, \eta=\eta_{r}$. According to Kolmogorov-Arnold-Moser (KAM) theory, under certain conditions [absence of resonances up to the 4-th order, non-zero coefficient in the normal form] the stationary point of the complete map is surrounded with a Cantor family of invariant curves (see, e.g. [5]). These latter conditions of KAM theory in the case under consideration can be written as one condition, namely $f\left(I_{r}, \eta_{r}\right) \neq 0$, where $f$ is a continuous function. We assume that the following condition is valid.

H2. There exist $I_{*}, \eta_{*}, \eta_{*}^{(1)}, \quad\left(\eta_{*}, \eta_{*}^{(1)}\right) \in L\left(I_{*}\right)$ such that $f\left(I_{*}, \eta_{*}\right) \neq 0$.

Then for $I$ close to $I_{*},\left|I-I_{*}\right|<c_{5}^{-1}$, there is a segment of the curve $L(I)$ where $\left|f\left(I, \eta, \eta^{(1)}\right)\right|>c_{6}^{-1}$. Without loss of generality one can assume that this segment coincides with $L(I)$ [otherwise, we can shorten $L(I)$ ]. According to KAM theory, for $\left|I_{r}-I_{*}\right|<c_{5}^{-1}$ there is an invariant curve at distance bigger than $c_{6}^{-1}$ from the point $\tilde{\xi}=0, \eta=\eta_{r}$. We call the domain surrounded by this curve a stability island of the Poincaré map. The area of this island is larger than $c_{7}^{-1}$. Returning from $\tilde{\xi}, \eta$ to the original variables $\xi, \eta$, we find that the area of the stability island is estimated from below as $c_{7}^{-1} \varepsilon$.

The boundary of the stability island (an invariant curve) is a section of an invariant torus on the energy level of the original system. The volume of the domain surrounded by this torus is larger than $C_{2}^{-1} \varepsilon$. The variation of $\hat{I}$ inside this domain is smaller than $C_{3} \varepsilon$.

\section{Distribution of periodic orbits}

Let $N\left(I_{c}, \Delta, \varepsilon\right)$ be the number of stable periodic orbits under consideration with $I^{(0)} \in$ $\left[I_{c}, I_{c}+\Delta\right] \subset \Xi_{0}$. According to Section 6.2 this number is equal to the number of "time" points $\xi \in\left[I_{c} / \varepsilon,\left(I_{c}+\Delta\right) / \varepsilon\right]$ when the point $\varepsilon^{-1} \Phi(\varepsilon \xi, \varepsilon)$ crosses the curve $\Lambda(\varepsilon \xi)$ on the torus $\mathbb{T}^{2}=\left\{\left(s_{1} \bmod 1, s_{2} \bmod 2\right)\right\}$. For values of $I_{c}$ such that $\gamma_{1}^{0}\left(I_{c}\right) / \gamma_{2}^{0}\left(I_{c}\right)$ is irrational one can calculate $\rho\left(I_{c}\right)=\lim _{\Delta \rightarrow 0} \lim _{\varepsilon \rightarrow 0}\left(\varepsilon N\left(h_{0}, I_{c}\right) / \Delta\right)$. We give the result omitting the standard argument based on ergodicity of winding of a torus with frequencies $\left(\gamma_{1}^{0}\left(I_{c}\right), \gamma_{2}^{0}\left(I_{c}\right)\right.$ ) (cf. [19]). Let $l$ be a natural parameter on $\Lambda\left(I_{c}\right)$, and $n\left(l, I_{c}\right)$ be a unit normal vector on $\Lambda\left(I_{c}\right)$. Introduce the notation

$$
\chi\left(I_{c}\right)=\int_{\Lambda\left(I_{c}\right)}\left|\left(\gamma^{0}\left(I_{c}\right), n\left(l, I_{c}\right)\right)\right| \mathrm{d} l,
$$

where $\left(\gamma^{0}, n\right)$ is the Euclidean scalar product of $\gamma^{0}$ and $n$. Hence, $\chi$ is an "absolute" phase flux of the vector field $\gamma^{0}$ across the curve $\Lambda$ : for the phase flux across any element of $\Lambda$ 
we take its absolute value. The value of $\chi\left(I_{c}\right)$ equals the product of the length of vector $\gamma^{0}$ and the full length of the projection of the curve $\Lambda\left(I_{c}\right)$ onto the normal to vector $\gamma^{0}$. Then

$$
\rho\left(I_{c}\right)=\frac{1}{2} \chi\left(I_{c}\right)
$$

(the coefficient $1 / 2$ in this formula appears because the surface area of the torus equals $2)$.

Let $\bar{N}\left(\Xi_{0}, \varepsilon\right)$ be the number of stable periodic orbits under consideration with $I^{(0)} \in \Xi_{0}$. If $\gamma_{1}^{0} \mathrm{~d} \gamma_{1}^{0} / \mathrm{d} I-\gamma_{2}^{0} \mathrm{~d} \gamma_{2}^{0} / \mathrm{d} I$ vanishes only on a set of measure 0 on $\Xi_{0}$, then

$$
\lim _{\varepsilon \rightarrow 0} \varepsilon \bar{N}\left(D_{0}, \varepsilon\right)=\frac{1}{2} \int_{\Xi_{0}} \chi(I) \mathrm{d} I
$$

\section{Acknowledgements}

The work was supported in part by grants RFBR 06-01-00117, 05-01-01119 and NSh1312.2006.1. C.S. has been supported by grants BFM2003-09504-C02-01, MTM200605849/Consolider (Spain) and CIRIT 2005 SGR-1028 (Catalonia).

\section{References}

[1] Büchner J , Zelenyi L M 1989 Regular and chaotic charged particle motion in magnetotail like field reversals, 1, Basic theory of trapped motion Journal of Geophysical Research 94(A9) 11821-11842

[2] Gurevich A V, Tsedilina E E Long distance propagation of Hf radio waves (Berlin, Springer-Verlag, 1985)

[3] Wisdom J 1985 A perturbative treatment of motion near the 3/1 commensurability Icarus 63(2) 272-289

[4] Arnold V I Mathematical methods of classical mechanics (Springer-Verlag, New York, 1978)

[5] Arnold V I , Kozlov V V and Neishtadt A I 1988 Mathematical aspects of classical and celestial mechanics (Encyclopedia of mathematical sciences 3) (Berlin: Springer)

[6] Arnold V I 1963 Small denominators and stability problems in classical and celestial mechanics Russ. Math. Surveys 1885

[7] Cary J R , Bruhwiller D S 1989 Diffusion of particles in a slowly modulated wave Physica D 40265

[8] Neishtadt A I and Sidorenko V V 2004 Wisdom system: dynamics in the adiabatic approximation, Celestial Mechanics and Dynamical Astronomy 90 307-330

[9] Elskens Y, Escande D F 1991 Slowly pulsating separatrices sweep homoclinic tangles where islands must be small: an extension of classical adiabatic theory Nonlinearity $4615-667$ 
[10] Neishtadt A I , Sidorenko V V and Treschev D V 1997 Stable periodic motions in the problem of passage through a separatrix Chaos 7 2-11

[11] Neishtadt A I , Sidorenko V V and Treschev D V 2001 On stability islands in the domain of separatrix crossings Nonlinear Mechanics ed V M Matrosov, V V Rumyantsev et al (Moscow: Fizmatlit) pp 192-203 (in Russian)

[12] Timofeev A V 1978 On the constancy of an adiabatic invariant when the nature of the motion changes Sov. Phys. JETP 48 656-659

[13] Cary J R , Escande D F and Tennyson J 1986 Adiabatic invariant change due to separatrix crossing Phys. Rev. A34 4256-4275

[14] Neishtadt A I 1986 Change of an adiabatic invariant at a separatrix Sov.J.Plasma Phys. 12 568-573

[15] Cary J R, Skodje R T 1989 Phase change between separatrix crossings Physica D $36287-316$

[16] Neishtadt A I 1987 On the change in the adiabatic invariant on crossing a separatrix in systems with two degrees of freedom PMM USSR 51 586-592

[17] Neishtadt A I and Vasiliev A A 2005 Phase change between separatrix crossings in slow-fast Hamiltonian systems Nonlinearity 18 1393-1406

[18] Neishtadt A I, Simó C, Treschev D V and Vasiliev A A 2006 Periodic orbits and stability islands in chaotic seas created by separatrix crossings in slow-fast systems, preprint, submitted to Discrete and Continuous Dynamical Systems

[19] Tal F A, Vanden-Eijnden E 2006 Transition state theory and dynamical corrections in ergodic systems Nonlinearity 19 501-509 